Supporting Information

\title{
Grain Boundary Defect Passivation of Triple Cation Mixed Halide Perovskite with Hydrazine-based Aromatic Iodide for Efficiency Improvement
}

Sheikh Ifatur Rahman ${ }^{1 \dagger}$, Buddhi Sagar Lamsallt, Ashim Gurung ${ }^{1 \dagger}$, Ashraful Haider

Chowdhury ${ }^{1}$, Khan Mamun Reza', Nabin Ghimire'1, Behzad Bahrami', Wenqin Luo²,

Raja Sekhar Bobba1', Jyotshna Pokharel', Abiral Baniya1, Ashiqur Rahman Laskar1, Khalid Emshadi', Md Tawabur Rahman', and Quinn Qiao1, 3*

1Department of Electrical Engineering, Center for Advanced Photovoltaics, South Dakota State University, Brookings 57007, SD, USA

2Department of Materials Chemistry, Huzhou University, Huzhou Zhejiang 313000, China

${ }^{3}$ Mechanical and Aerospace Engineering, Syracuse University, Syracuse, NY 13244 “Corresponding author: Email: qiquan.qiao@sdstate.edu; quqiao@syr.edu †These authors equally contributed to this work. 
a)

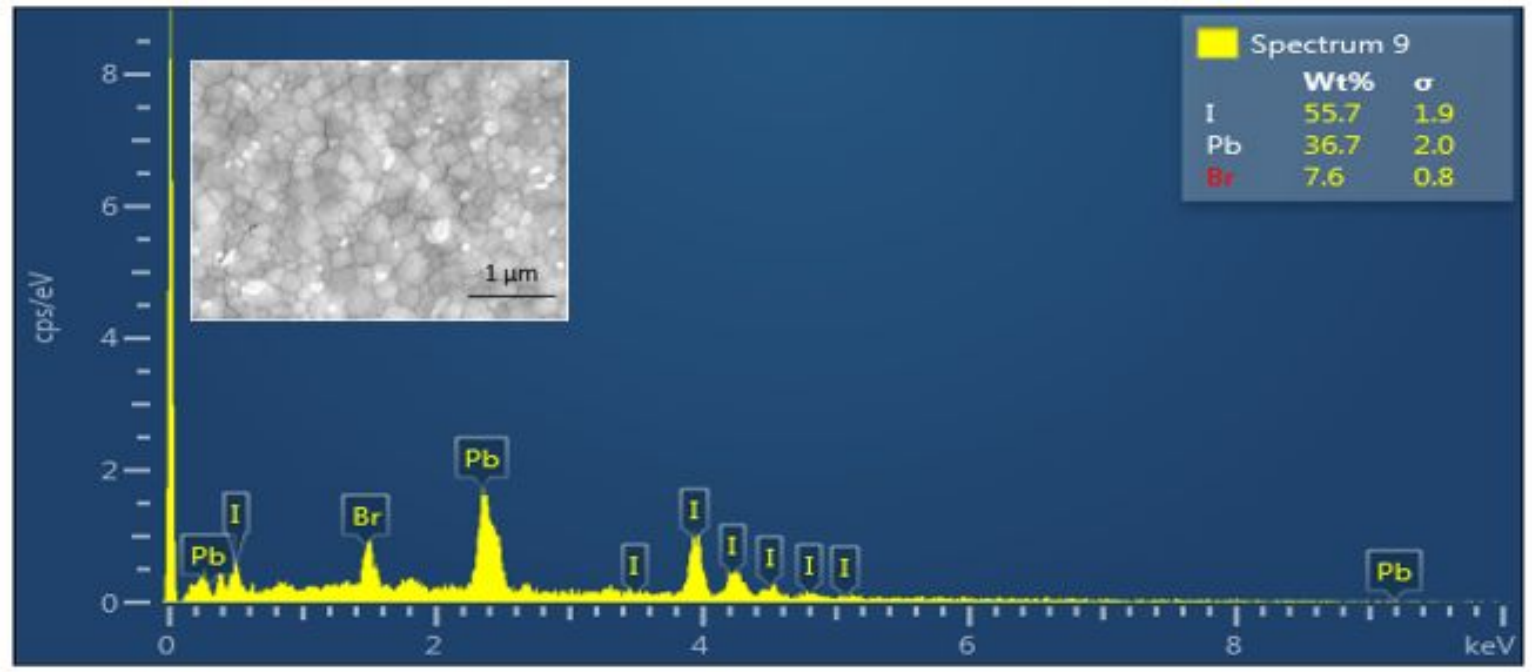

b)

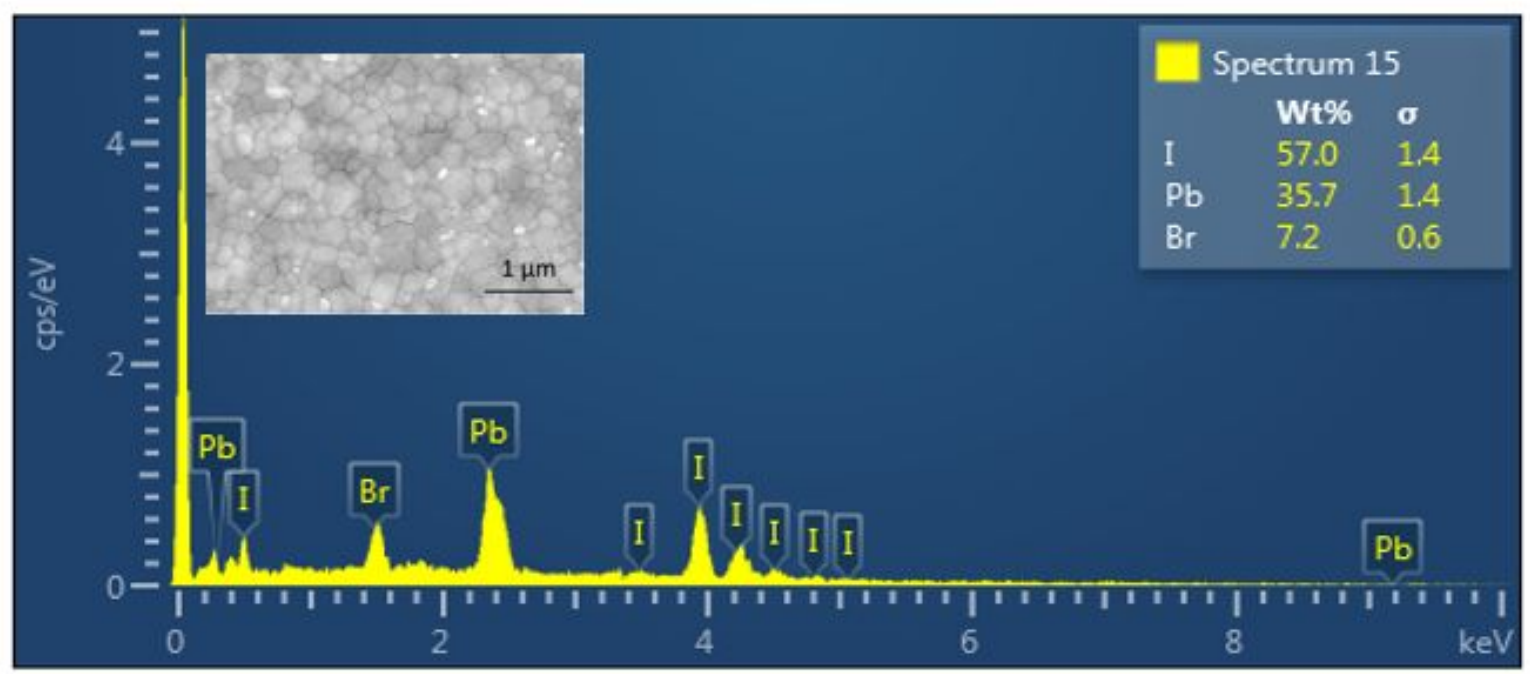

Figure S1. EDX results of a) control (untreated) and b) $2 \mathrm{mg} / \mathrm{ml}$ PHI-treated perovskite films. 


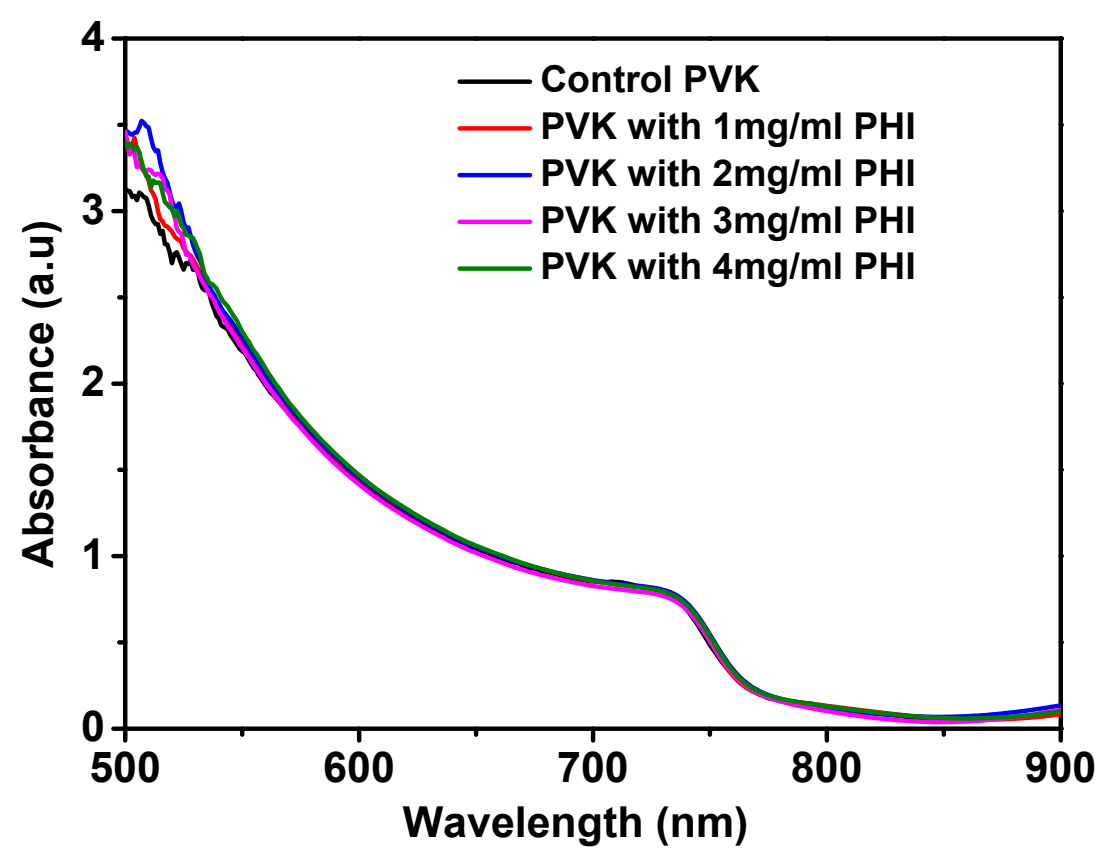

Figure S2. Ultraviolet-visible absorption of $\left(\mathrm{FA}_{0.83} \mathrm{MA}_{0.17}\right)_{0.95} \mathrm{Cs}_{0.05} \mathrm{~Pb}\left(\mathrm{I}_{0.83} \mathrm{Br}_{0.17}\right)_{3}$ perovskite solar cells without and with perovskite layer modification. The band gap remains unchanged $\sim 1.59 \mathrm{eV}$. Absorption of the structure remained similar in all conditions. 
a)

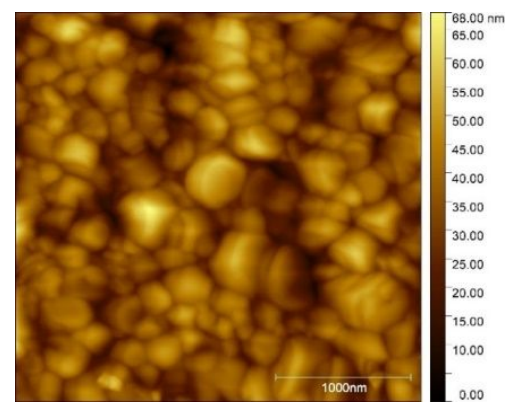

c)

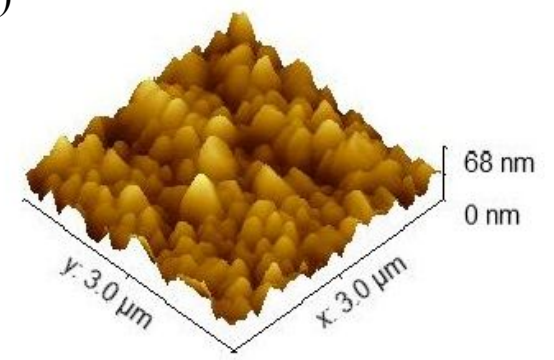

b)

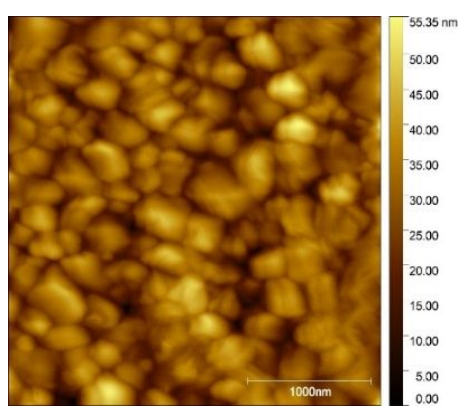

d)

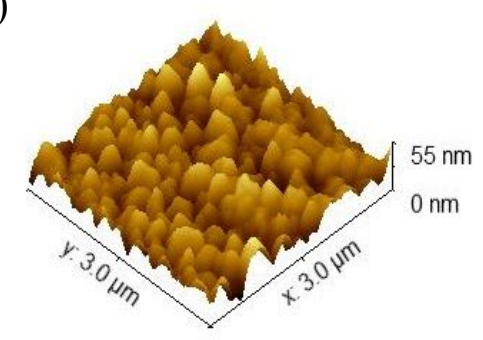

Figure S3. Atomic force microscopy (AFM) images of perovskite films without and with PHI treatment. a), c) AFM of perovskite film without PHI treatment and b), d) with $2 \mathrm{mg} / \mathrm{ml}$ PHI treatment. After PHI treatment, the roughness of perovskite layer reduced from $34 \mathrm{~nm}$ to 28.9 $\mathrm{nm}$ indicating smoother perovskite layer for better charge transport. 
The band bending and energy diagram on the basis of the KPFM studies are depicted in Fig. S4. As we can see from the band bending at the GBs of the perovskite layer (Fig. S4a), an electron barrier exists at the GBs for the passivated perovskite film while, a hole barrier exists for the control or unpassivated film. This phenomenon can be coupled to the interface of the perovskite with the HTM (Spiro-OMeTAD) as shown in Figure S4b,c. It is favorable to have an electron barrier at the GBs of the perovskite at the interface with the HTM (as observed for the passivated perovskite (Fig. S4c), as it would facilitate efficient positive charge carriers (holes) to the HTM, while blocking electron transfer that reduces charge recombination. In contrary, the hole barrier observed in the case of control perovskite hinders the efficient hole transfer at the GB interface with the HTM (Fig. S4b).

a)

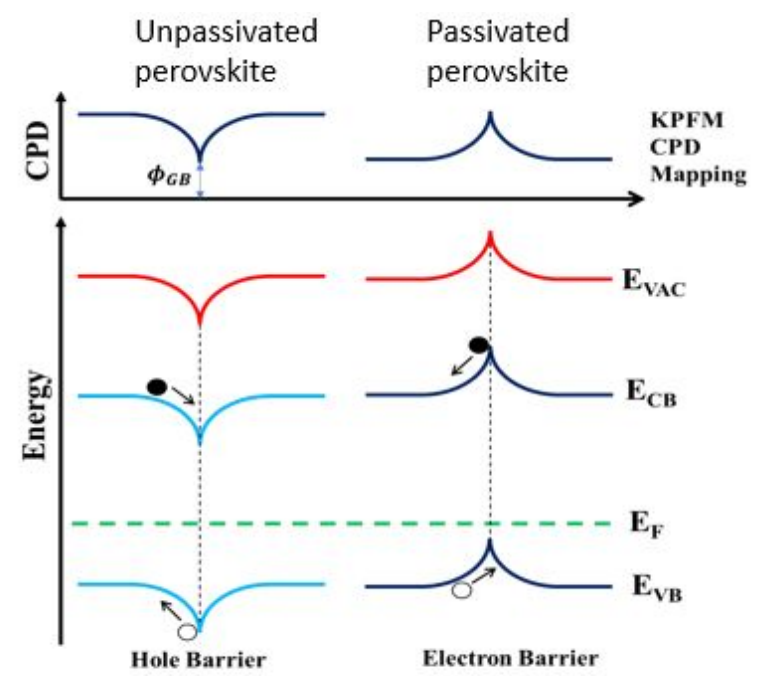

b)

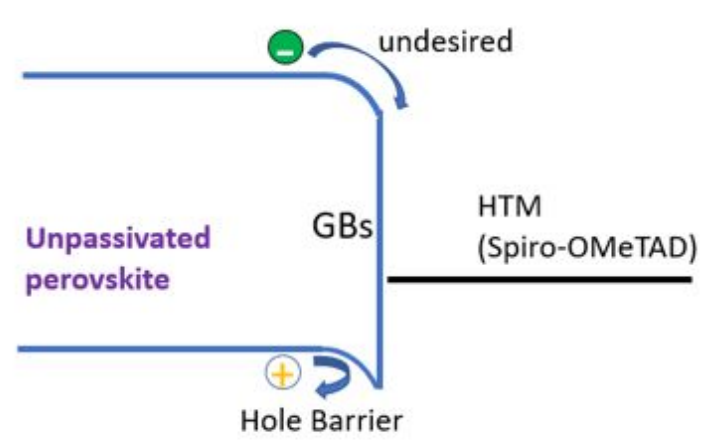

Unfavorable band bending at the GB interface with HTM c)

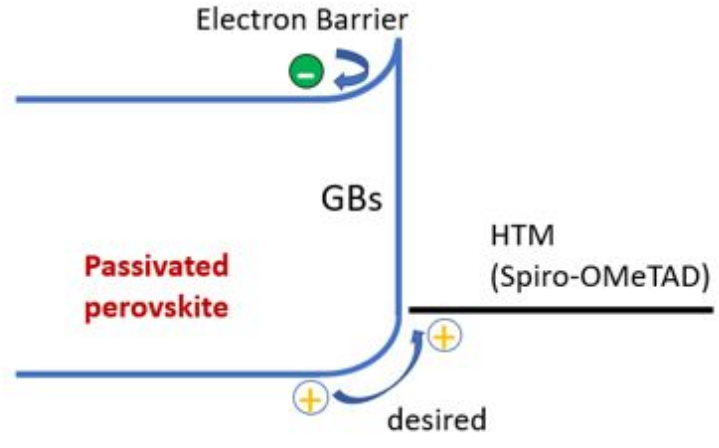

Favorable band bending at the GB interface with HTM

Figure S4. Schematic showing band diagrams based on the CPD mapping obtained from KPFM measurements (a) at the GB in the perovskite film; $(b, c)$ at the perovskite/HTM interface with (b) control (unpassivated) and (c) passivated perovskite film. 




Figure S5. Current-Voltage $(\mathrm{J}-\mathrm{V})$ characteristics in different PHI concentration. J-V curve from forward scan (0-1.2 V) and reverse scan (1.2-0 V) are both measured.

Table S1. Device performance summary in different PHI concentration.

\begin{tabular}{|c|c|c|c|c|c|c|c|}
\hline $\begin{array}{c}\text { PHI } \\
\text { Concentration } \\
(\mathbf{m g} / \mathbf{m l})\end{array}$ & $\begin{array}{c}\text { Scan } \\
\text { Direction }\end{array}$ & $\begin{array}{l}\mathbf{V}_{\text {OC }} \\
(\mathrm{V})\end{array}$ & $\begin{array}{c}\mathbf{J}_{\mathrm{SC}} \\
\left(\mathrm{mA} / \mathrm{cm}^{2}\right)\end{array}$ & $\begin{array}{c}\text { FF } \\
(\%)\end{array}$ & $\begin{array}{c}\text { PCE } \\
(\%)\end{array}$ & Average & $\begin{array}{c}\text { H- } \\
\text { index }\end{array}$ \\
\hline \multirow{2}{*}{$\mathbf{0}$} & $\mathrm{RS}$ & 1.11 & 21.71 & 0.72 & 17.43 & \multirow{2}{*}{$16.39 \%$} & \multirow{2}{*}{$11.93 \%$} \\
\hline & FS & 1.09 & 21.73 & 0.65 & 15.35 & & \\
\hline \multirow{2}{*}{1} & $\mathrm{RS}$ & 1.13 & 23.96 & 0.68 & 18.46 & \multirow{2}{*}{$18.04 \%$} & \multirow{2}{*}{$4.55 \%$} \\
\hline & FS & 1.11 & 23.69 & 0.67 & 17.62 & & \\
\hline \multirow{2}{*}{2} & $\mathrm{RS}$ & 1.13 & 24.49 & 0.75 & 20.62 & \multirow{2}{*}{$20.34 \%$} & \multirow{2}{*}{$2.76 \%$} \\
\hline & FS & 1.13 & 24.30 & 0.73 & 20.05 & & \\
\hline \multirow{2}{*}{3} & $\mathrm{RS}$ & 1.14 & 23.4 & 0.66 & 17.71 & \multirow{2}{*}{$17.26 \%$} & \multirow{2}{*}{$5.02 \%$} \\
\hline & FS & 1.13 & 23.26 & 0.64 & 16.82 & & \\
\hline \multirow{2}{*}{4} & $\mathrm{RS}$ & 1.11 & 22.28 & 0.69 & 17.15 & \multirow{2}{*}{$16.80 \%$} & \multirow{2}{*}{$4.02 \%$} \\
\hline & $\mathrm{FS}$ & 109 & 22.32 & 068 & 1646 & & \\
\hline
\end{tabular}

$\mathrm{H}$-index $=\left(\mathrm{PCE}_{\text {reverse }}-\mathrm{PCE}_{\text {forward }}\right) / \mathrm{PCE}_{\text {reverse }}$ 


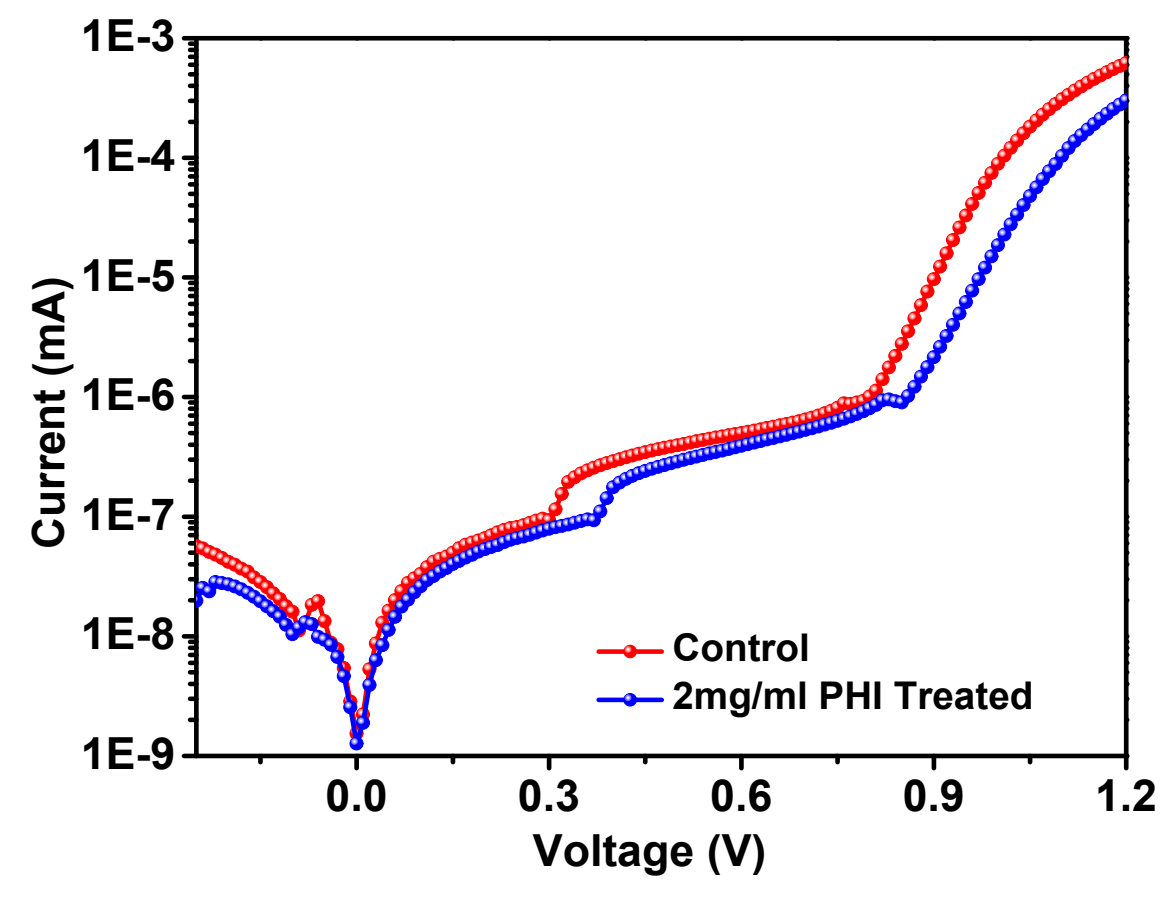

Figure S6. Dark Current-Voltage (J-V) characteristics in different PHI concentration. J-V curve from $-0.3 \mathrm{~V}$ to $1.2 \mathrm{~V}$. 

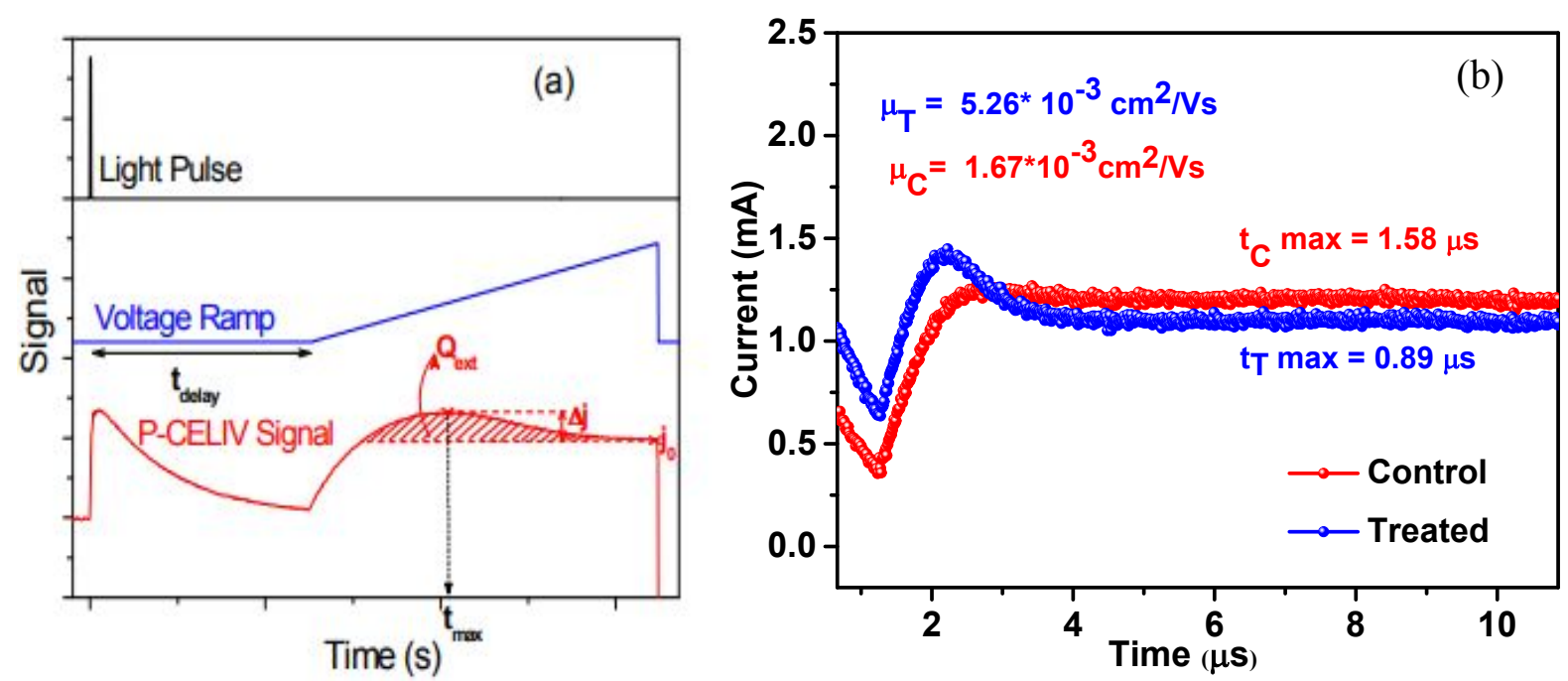

Figure S7. (a) Photo-CELIV measurement mechanism. ${ }^{1}$ (b)Photo-CELIV measurements to calculate the mobility for the control perovskite and treated perovskite devices.

The charge carrier mobilities were calculated using the equation $\mu=\frac{K^{2} * 2 * d^{2}}{A * t_{\max }}$ where $\mathrm{K}$ is the correction factor and the value of $k=\frac{2}{3}, \mathrm{~d}$ is the active layer thickness in $\mathrm{cm}, \mathrm{A}$ is the voltage ramp rate in $\mathrm{V} / \mathrm{Hz}, t_{\max }$ is the maximum time for charge extraction. ${ }^{1}$

Table S2. Charge Carrier Mobility Measurements

\begin{tabular}{|c|c|c|c|c|}
\hline & $t_{\max }(\boldsymbol{\mu s})$ & $\begin{array}{c}\text { Mobility } \\
\left(\mathbf{c m}^{2} / \mathbf{V s}\right)\end{array}$ & Thickness(nm) & Voltage Ramp Rate \\
\hline Control & 1580 & $1.67^{*} 10^{-3}$ & 500 & $4 * 10^{5}$ \\
\hline Treated & 890 & $5.26^{*} 10^{-3}$ & 500 & $4 * 10^{5}$ \\
\hline
\end{tabular}


(a)

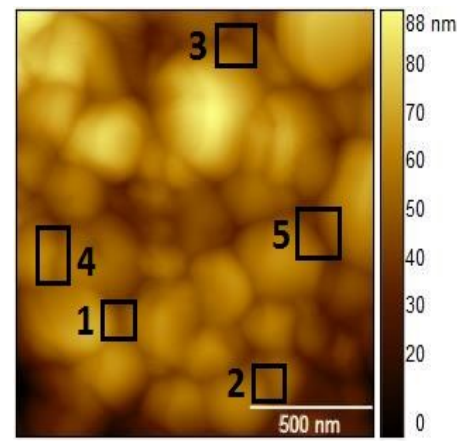

(d)

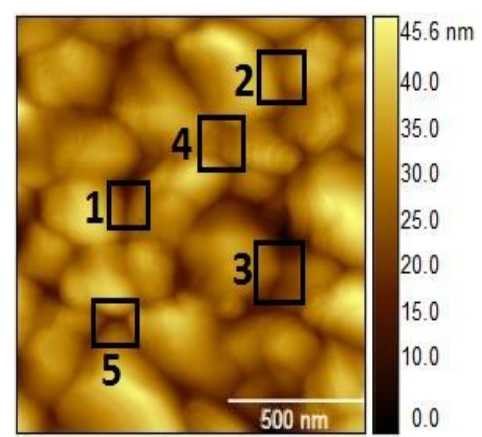

(b)

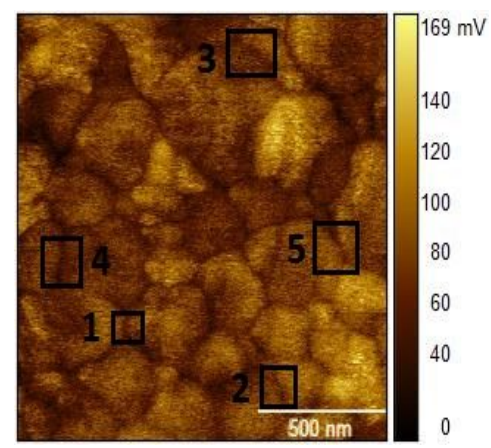

(e)

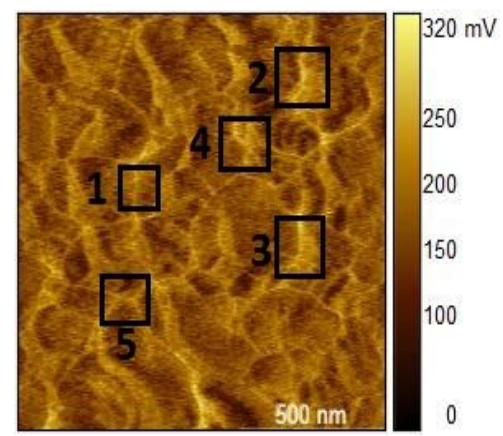

(c)

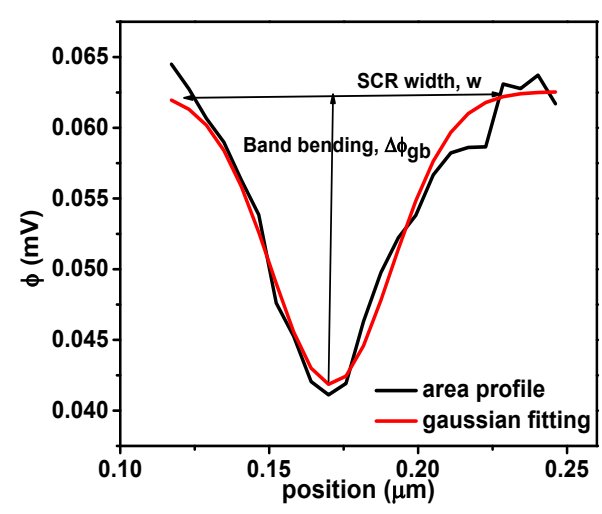

(f)

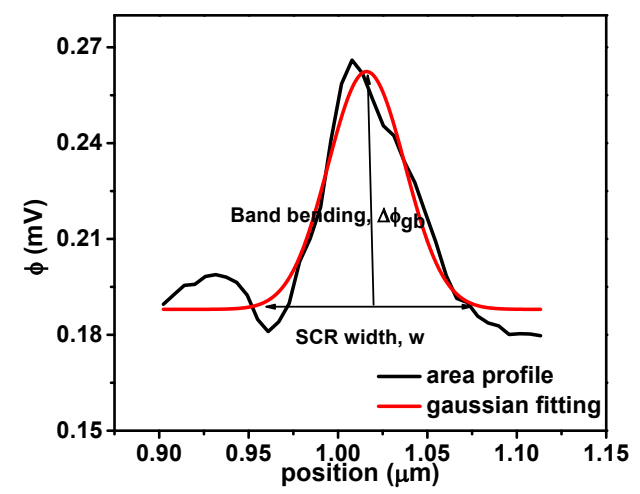

Figure S8. Trap state density calculation: a) AFM-topography image of control perovskite film,

b) KPFM mapping of control perovskite film, c) trap state density measurement using area profile for control perovskite, d) AFM-topography image of perovskite film treated with PHI, e) KPFM mapping of perovskite film treated with PHI, f) trap state density calculation of perovskite film treated with PHI from area profile.

\section{Density of Trap State Calculation ${ }^{2}$ :}

By assuming that the grain boundary corresponds to a surface with a surface charge we can determine the net doping $P_{n e t}$ of the absorber material from the size of the band bending $\Delta \phi_{g b}$ and the SCR width $w$,

$$
P_{n e t}=\frac{2 \epsilon_{0} \epsilon \Delta \phi_{g b}}{e^{2} w^{2}}
$$

where, $e$ is the elementary charge, $\epsilon_{0}$ is the permittivity of free space and $\epsilon$ is the dielectric constant of the perovskite. By the fact that the SCR width at the grain boundaries does not 
extend to the center of the grains, we can estimate the density of charged trap states $P_{g b}$, following a model originally proposed for transport across grain boundaries in polycrystalline silicon,

$$
P_{g b}=\frac{1}{e} \sqrt{8 \epsilon \epsilon_{0} P_{n e t} \Delta \phi_{g b}} \ldots \ldots
$$

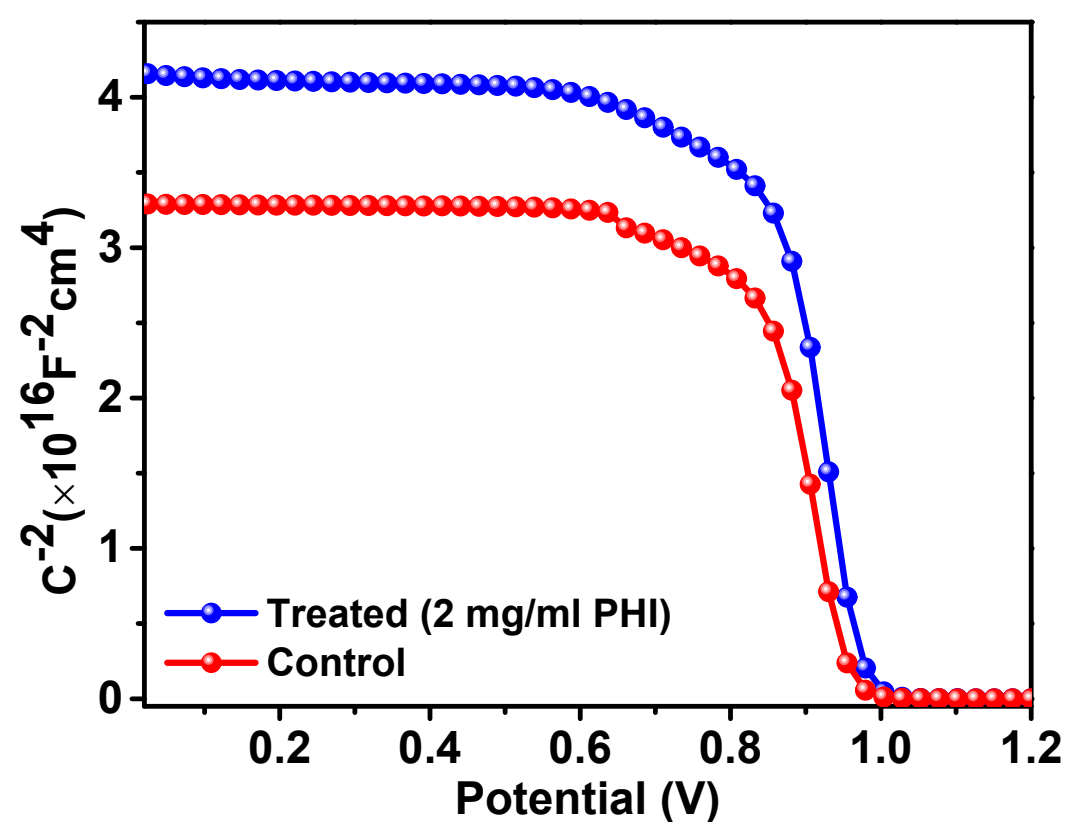

Figure S9. Mott-Schottky plot for the control and treated perovskite devices.

Table S3. Flat Band Potential and Slope obtained from Mott Schottky Plot

\begin{tabular}{|c|c|c|}
\hline & Slope & $\mathbf{V}_{\mathbf{b i}}$ \\
\hline Control & $2.34 * 10^{16}$ & 0.96 \\
\hline Treated & $2.88 * 10^{16}$ & 1.00 \\
\hline
\end{tabular}



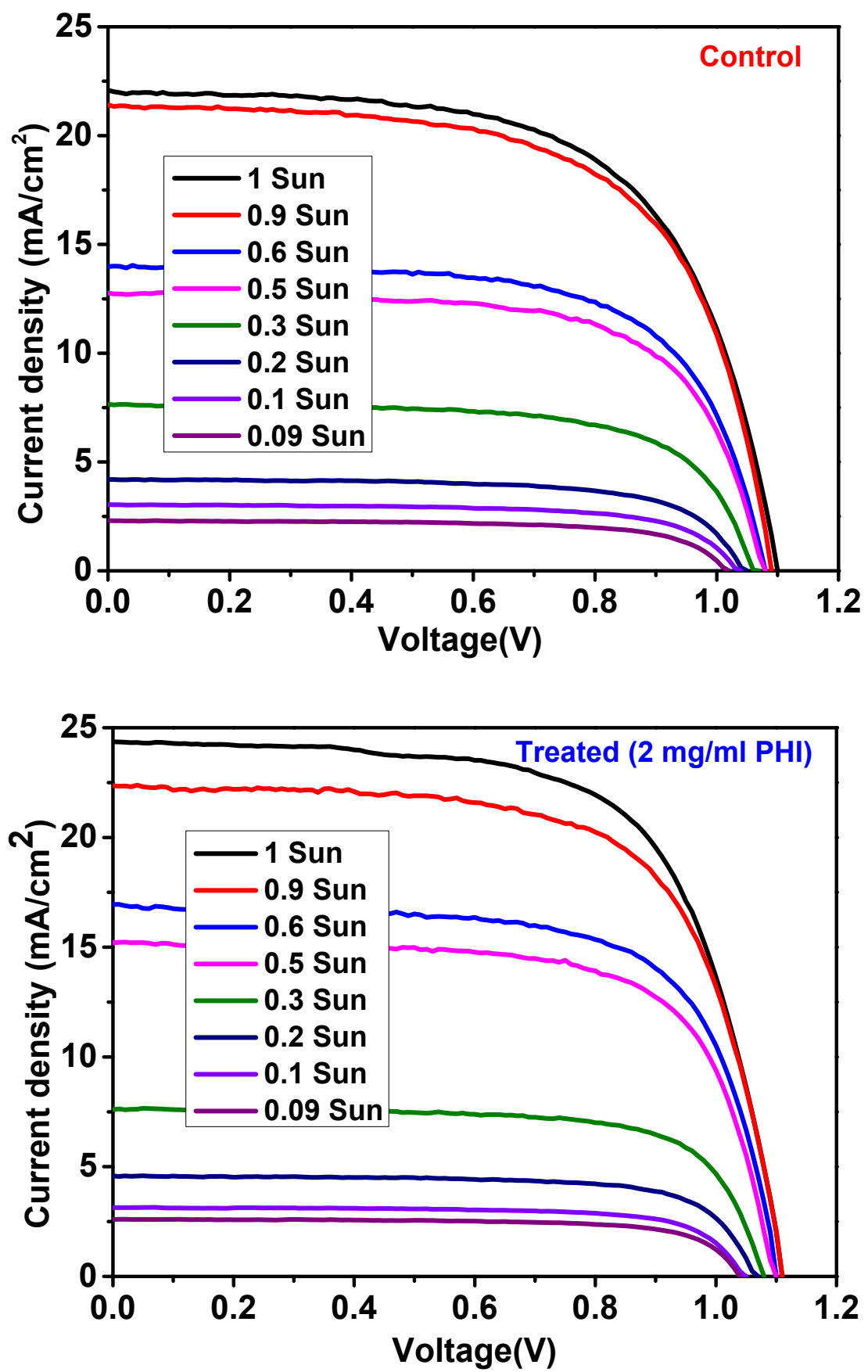

Figure S10. J-V curve of the device without PHI treatment and with PHI treatment under different light intensity ranging from 1 Sun to 0.09 Sun 

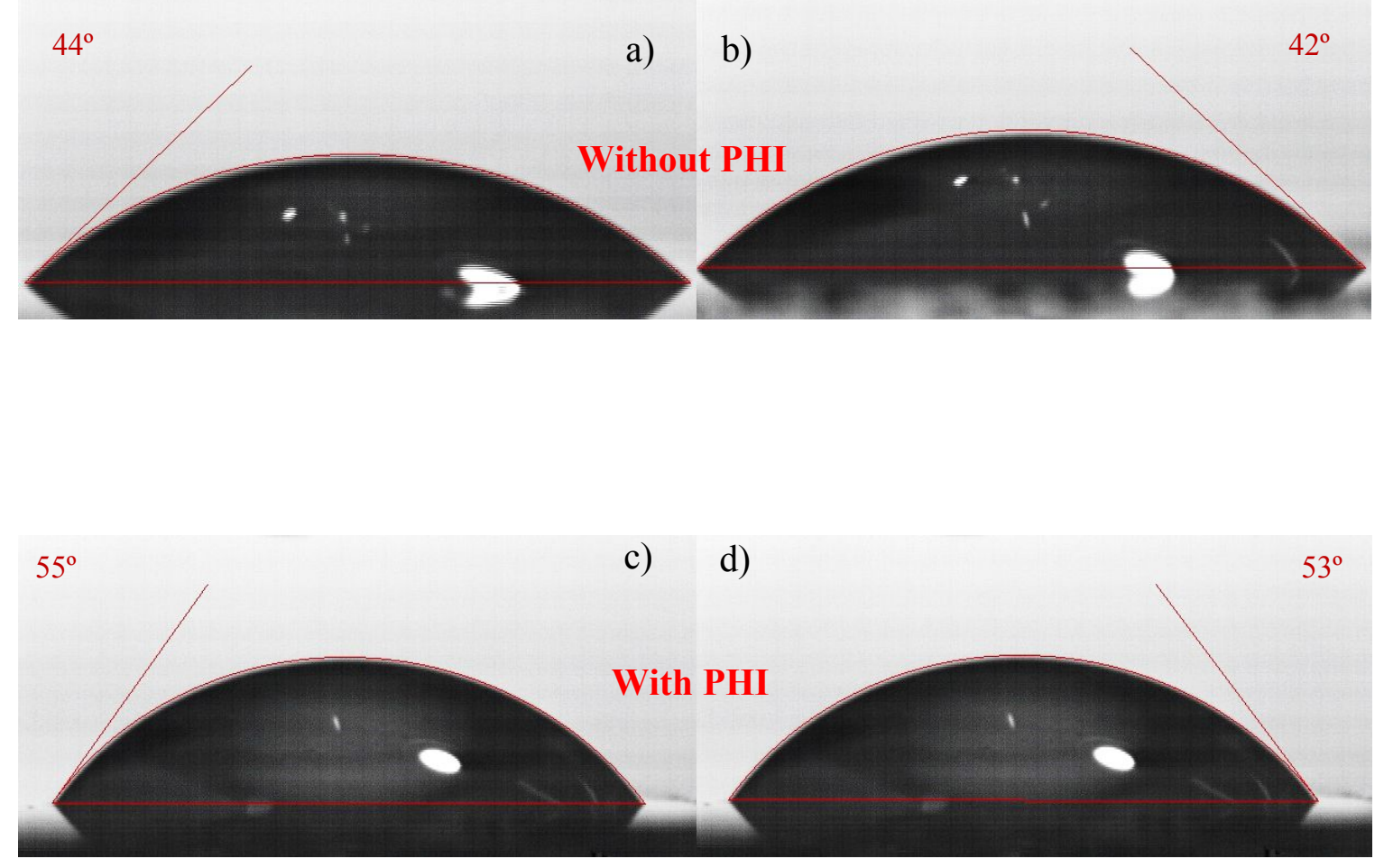

Figure S11. Contact angle measurements of $(a, b)$ control perovskite film and (c, d)perovskite film treated with $2 \mathrm{mg} / \mathrm{ml} \mathrm{PHI}$. 

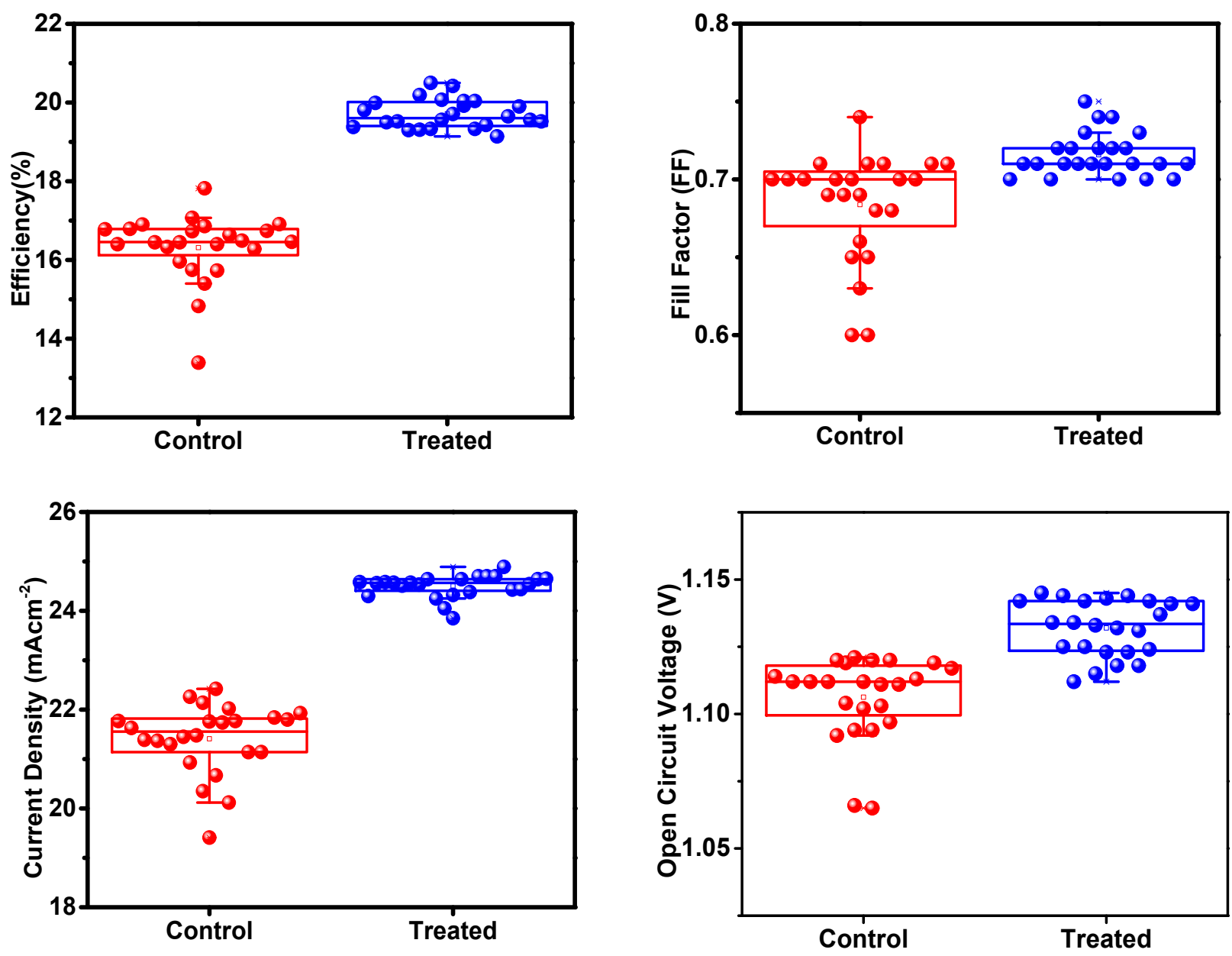

Figure S12. Statistical Analysis of device parameters

Table S4. Statistical Data of Control and Treated Devices

\begin{tabular}{|c|c|c|c|c|}
\hline & Voc $(\mathbf{V})$ & Jsc $\left(\mathbf{m A} / \mathbf{c m}^{2}\right)$ & FF & Efficiency (\%) \\
\hline Control & $1.106 \pm 0.016$ & $21.410 \pm 0.69$ & $0.683 \pm 0.03$ & $16.315 \pm 0.85$ \\
\hline Treated & $1.132 \pm 0.010$ & $24.501 \pm 0.22$ & $0.715 \pm 0.01$ & $19.713 \pm 0.36$ \\
\hline
\end{tabular}




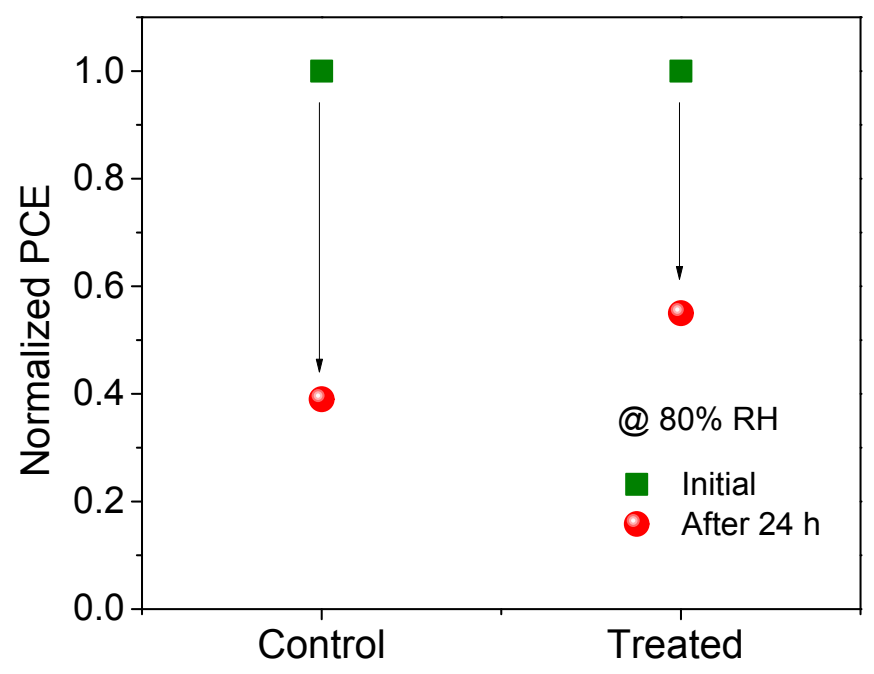

Figure S13. Stability tests of the control and treated devices at a high relative humidity of $80 \%$ stored for 24 hours. The devices were unencapsulated for this test.

\section{References}

1. Venkatesan, S.; Ngo, E. C.; Chen, Q.; Dubey, A.; Mohammad, L.; Adhikari, N.; Mitul, A. F.; Qiao, Q., Benzothiadiazole-based Polymer for Single and Double Junction Solar Cells with High Open Circuit Voltage. Nanoscale 2014, 6 (12), 7093-7100.

2. Sadewasser, S.; Glatzel, T.; Schuler, S.; Nishiwaki, S.; Kaigawa, R.; Lux-Steiner, M. C., Kelvin Probe Force Microscopy for the Nano Scale Characterization of Chalcopyrite Solar Cell Materials and Devices. Thin Solid Films 2003, 431, 257-261. 